\title{
Analyse d'une défaillance par voilement global au cours du rotomoulage d'un produit tri-couche
}

\author{
Hatem Ghazel ${ }^{a}$, Abdelkarim Ben Rhima et Mohamed Jemmali \\ Unité de recherche en mécanique appliquée, École Nationale d’Ingénieurs de Tunis, BP 37, Le Belvédère, 1002 Tunis, Tunisie
}

Reçu le 31 juillet 2009, accepté le 17 janvier 2011

\begin{abstract}
Résumé - Pour situer l'origine d'une anomalie récurrente sur une coque en sandwich tri-couche rotomoulé, une analyse d'instabilité par voilement global est développée. Cette voie est suggérée par la caractérisation de la géométrie du défaut observé et par l'imprédictibilité de son apparition. Le problème thermomécanique est posé dans un cadre non-linéaire en se restreignant à l'évolution au refroidissement en phase solide après que la fraction cristallisée ait atteint le niveau de saturation. Dans le modèle proposé, les non-linéarités liées à l'évolution des propriétés matérielles en fonction de la température et celles associées au contact unilatéral moule/pièce sont considérées. La résolution du problème thermomécanique instationnaire est réalisée par la méthode des éléments-finis au moyen du code Abaqus/Explicit. Le recalage de l'évolution thermique est conduit par un schéma de prédiction-correction de la conductance sur l'interface de contact. Pour l'analyse de la stabilité sous l'effet du chargement thermomécanique, nous appliquons la méthode de perturbation linéaire et les résultats de simulation confirment bien la nature de défaillance suspectée. Les deux premiers modes de voilement calculés sont en bon accord avec les observations dans les conditions réelles du processus.
\end{abstract}

Mots clés : Rotomoulage / tri-couche / thermomécanique / évolution / voilement

\begin{abstract}
Investigation on a global buckling defect during the rotomolding of a three-layer hull. To investigate the origin of a recurring anomaly that affects a rotomolded three-layer sandwich hull, we prospect numerically a way of instability by global buckling. Such alternative is suggested, on one hand by the final geometry at the return to the room temperature and, on the other hand by the unpredictable occurring of the anomaly. The thermo mechanical problem is set within a nonlinear framework and limited to the cooling sequence in solid phase after the degree of crystallinity has reached its saturation level. The non-linearities of the material are introduced through temperature-dependent evolutions and the geometrical ones at the interface mould/hull are all over taken into account through unilateral contact conditions. The computational of the unsteady thermo mechanical problem is carried out by means of the finite-element code Abaqus/Explicit and the thermal evolution adjustment is performed by a predictioncorrection scheme for the gap conductance. To achieve the buckling analysis under thermo mechanical loading we make use of the linear perturbation method and the results we obtain are consistent with the defect so far suspected. Thus, the two first buckling modes computed are in a good agreement with the defects under the real conditions of the process.
\end{abstract}

Key words: Rotomolding / three-layer / thermo mechanics / evolution / buckling

\section{Introduction}

Le procédé de rotomoulage $[1,2]$ est avantageusement mis en œuvre pour l'obtention de coques minces en sandwiches tri-couche offrant un rapport de performance masse/rigidité élevé [3]. Ceci tient notamment à la continuité matérielle assurée entre les différentes couches

\footnotetext{
${ }^{a}$ Auteur pour correspondance : ghazel.hatem@hotmail.fr
}

au cours des trois séquences de cuisson et de la séquence de refroidissement pendant laquelle la cristallisation se produit [4].

Bien que la séquence de cuisson continue à faire l'objet d'une recherche intensive traitant de multiples volets thermo-physiques $[5,6]$ la littérature reste peu fournie au sujet de la séquence de refroidissement avec un intérêt limité pour l'essentiel à l'évolution thermique $[7,8]$. Plus particulièrement, les produits tri-couche en polyéthylène 


\section{Nomenclature}

\begin{tabular}{|c|c|c|}
\hline$d$ & distance & $(\mathrm{m})$ \\
\hline$[\mathrm{C}]$ & matrice de capacitance & \\
\hline$C_{\mathrm{p}}$ & chaleur spécifique à pression constante & $\left(\mathrm{J} . \mathrm{kg}^{-1} \cdot \mathrm{K}^{-1}\right)$ \\
\hline$c$ & constante & \\
\hline$E$ & module de Young & $(\mathrm{MPa})$ \\
\hline$f$ & coefficient de frottement de glissement & \\
\hline$h$ & coefficient de convection & $\left(\mathrm{W} \cdot \mathrm{m}^{-2} \cdot \mathrm{K}\right)$ \\
\hline$H$ & fonction échelon de Heaviside & \\
\hline$I$ & tenseur identité & \\
\hline$k$ & conductivité thermique & $\left(\mathrm{W} \cdot \mathrm{m}^{-1} \cdot \mathrm{K}^{-1}\right)$ \\
\hline$[\mathrm{K}]$ & matrice de rigidité & \\
\hline$m$ & constante & \\
\hline$[\mathrm{M}]$ & matrice de masse & \\
\hline$N u$ & nombre de Nusselt & \\
\hline $\operatorname{Pr}$ & nombre de Prandtl & \\
\hline $\mathcal{R}(\mathrm{O}, \boldsymbol{x}, \boldsymbol{y}, \boldsymbol{z})$ & repère lié au système & \\
\hline$R e$ & nombre de Reynolds & \\
\hline$R_{\mathrm{t}}$ & rugosité totale & $(\mu \mathrm{m})$ \\
\hline$t$ & temps & $(\mathrm{s})$ \\
\hline$T$ & température & $\left({ }^{\circ} \mathrm{C}\right)$ \\
\hline$u$ & champ de vecteur de déplacement & \\
\hline$\dot{\boldsymbol{u}}_{i / j}$ & champ de vecteur de vitesse de glissement & \\
\hline $\boldsymbol{x}$ & vecteur position & \\
\hline$\left(x_{1}, x_{2}, x_{3}\right)$ & coordonnées locales & \\
\hline$x_{3}$ & coordonnée normale extérieure & $(\mathrm{m})$ \\
\hline \multicolumn{3}{|l|}{ Lettres grecques } \\
\hline$\alpha$ & coefficient de dilatation thermique isotrope & $\left(\mathrm{K}^{-1}\right)$ \\
\hline$\varepsilon$ & champ tenseur de déformation & \\
\hline$\varepsilon^{3}$ & espace affine euclidien & \\
\hline$\lambda$ & coefficient de Lamé & \\
\hline$\lambda_{c}$ & valeur propre & \\
\hline$\mu$ & coefficient de Lamé & $(\mathrm{MPa})$ \\
\hline$\nu$ & coefficient de Poisson & \\
\hline$\theta$ & ecart de température & $(\mathrm{K})$ \\
\hline$\rho$ & masse volumique & $\left(\mathrm{kg} \cdot \mathrm{m}^{-3}\right)$ \\
\hline$\sigma$ & champ de tenseur de contrainte de Cauchy & \\
\hline$\Omega$ & domaine & \\
\hline$\partial \Omega$ & frontière d'un domaine & \\
\hline$\partial \Omega_{k-k+1}$ & interface $\Omega_{k} \cap \Omega_{k+1}$ & \\
\hline$\dot{\omega}, \dot{\phi}$ & vecteur vitesse & $\left(\mathrm{s}^{-1}\right)$ \\
\hline$\psi, \phi$ & vitesses angulaires & $\left(s^{-1}\right)$ \\
\hline
\end{tabular}

n'ont pas encore fait l'objet de travaux de modélisation à même de permettre l'explication de certaines anomalies rencontrées par les rotomouleurs. En effet, les structures minces obtenues par rotomoulage continuent à poser des difficultés notamment par la manifestation de déformations dont les modes et les causalités demeurent mal cernées. Il s'ensuit que le réglage du cycle de production fait souvent appel à l'empirisme.

Nous nous attachons dans cette contribution à une étude de cas sur la production de coques de canoë kayaks affectées par la manifestation d'une déformation globale à la fois récurrente et imprédictible et qui est observée à un stade avancé de la séquence de refroidissement. Il s'agit d'une déformation de forte amplitude (Fig. 1) avec gauchissement du bordé de coque qui se produit au voisinage de l'étrave. Ces déformations constituent un défaut qui pénalise la qualité de la pièce finie.

L'objectif visé est d'identifier les causes de ce défaut en prospectant une voie d'instabilité par voilement global. L'étude est initiée par une caractérisation géométrique du défaut observé afin d'orienter vers une voie de modélisation. Un modèle numérique est ensuite proposé pour confirmer notre suspicion d'une défaillance par instabilité par voilement global.

À cet effet, nous posons d'abord une formulation instationnaire non linéaire qui fournit l'histoire thermomécanique lors du refroidissement. Cette restriction à 


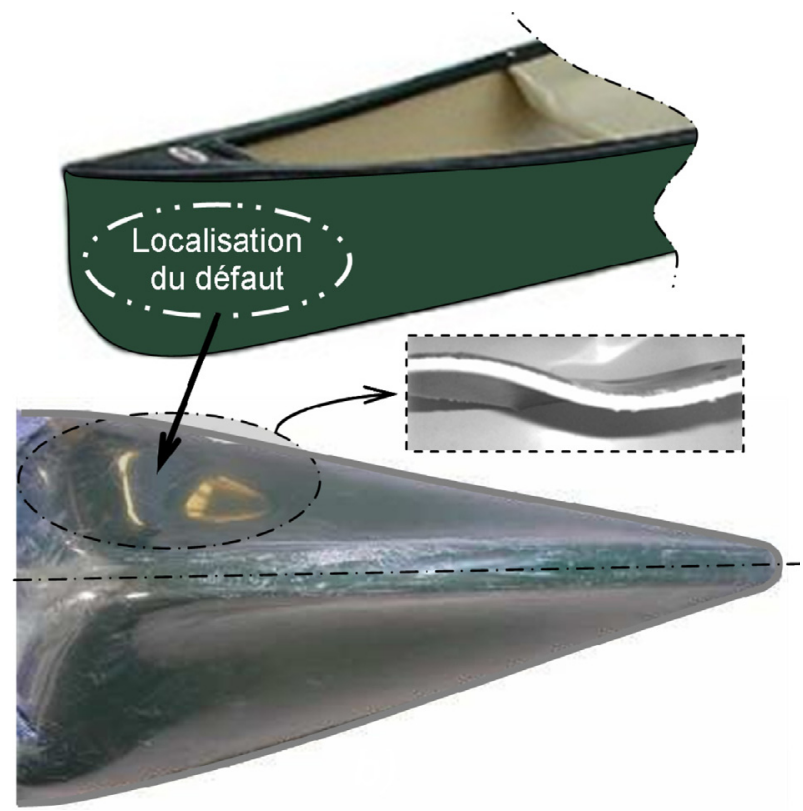

Fig. 1. Localisation et morphologie du défaut observé.

la séquence de refroidissement se justifie, d'une part par l'oubli de l'histoire thermomécanique lors de la transition de l'état amorphe à l'état cristallin, et d'autre part par le retrait non isotrope de la pièce et les décollements qu'il peut engendrer. Nous procédons ensuite à la résolution numérique du problème par la méthode des éléments-finis (MEF) et discutons enfin les résultats obtenus.

\section{Caractérisation géométrique}

La paroi tri-couche est constituée par une peau extérieure en polyéthylène haute densité (PEHD), une âme en mousse de polyéthylène et une peau intérieure en polyéthylène basse densité (PEBD). Les peaux PEHD et PEBD appliquées en couches minces sont assez uniformes avec respectivement $2 \div 3 \mathrm{~mm}$, et $1 \div 2 \mathrm{~mm}$ d'épaisseur. Par contre, la mousse a une épaisseur importante de l'ordre de $80 \mathrm{~mm}$ au niveau de l'étrave et s'amincit par ailleurs pour se stabiliser autour d'une épaisseur de $7 \div 9 \mathrm{~mm}$ sur les bordés de coque. Cette répartition des épaisseurs est précisée sur la figure 2 .

Pour poser un cadre de modélisation du problème, une méthodologie déductive des relations de causes à effets est retenue en adoptant pour observable d'entrée la géométrie actuelle de la zone déformée après retour à la température ambiante. À cet effet, des échantillons représentatifs indépendants sont prélevés sur deux coques déformées et un scannage mécanique est appliqué pour disposer d'un nuage de 916 points. Les surfaces ainsi numérisées sont maillées puis exportées vers le code EF Cast3M [9] en vue de leur post-traitement.

La cartographie de la composante normale du déplacement dans un panneau de $156 \times 252 \mathrm{~mm}$ (Fig. 3) montre que les amplitudes sont importantes et atteignent

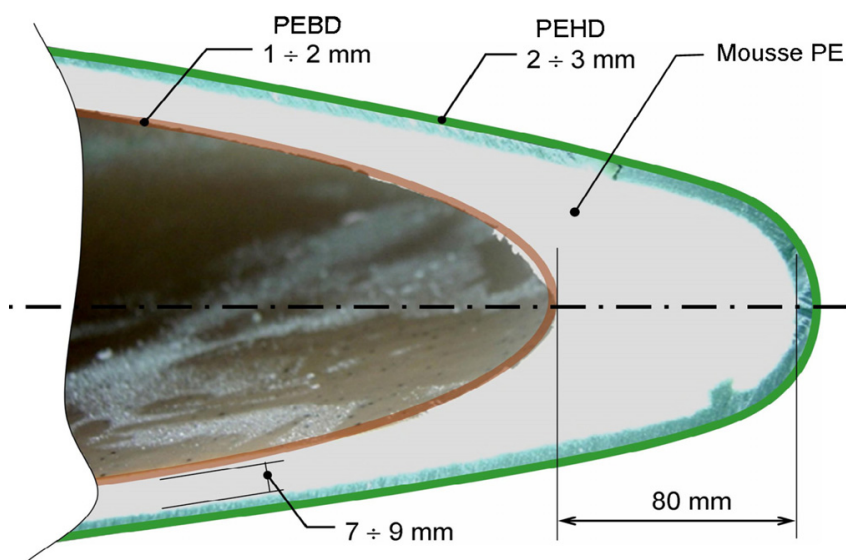

Fig. 2. Constitution et épaisseurs de la coque.

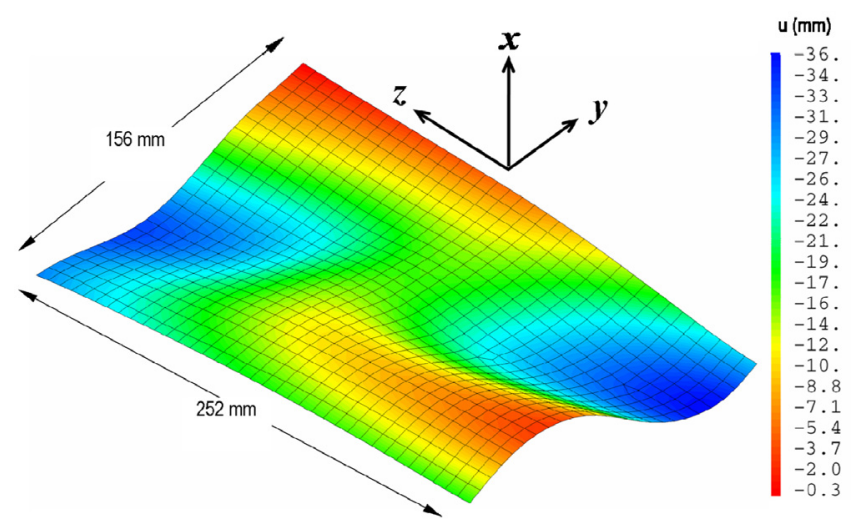

Fig. 3. Composante normale mesurée du déplacement résiduel.

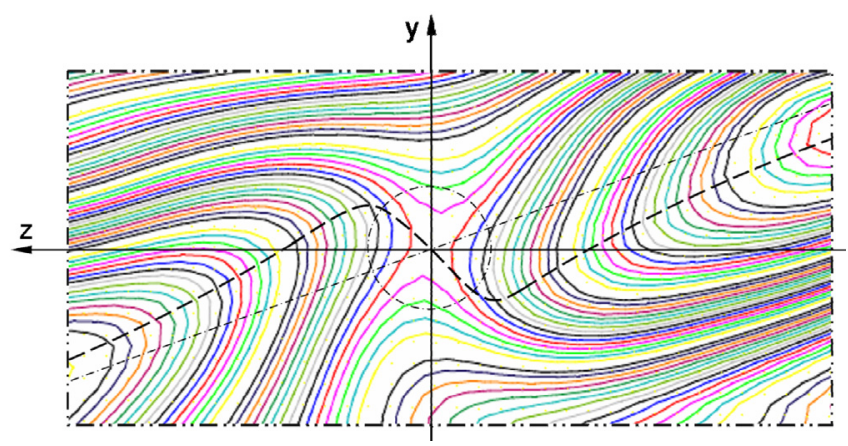

Fig. 4. Antisymétrie des lignes de niveaux du déplacement normal.

en certains lieux $36 \mathrm{~mm}$ ce qui suggère un caractère incontrôlé de la déformation. Par ailleurs, la projection cartographique des lignes de niveaux sur le plan $(y, z)$ tangent au centre du panneau (Fig. 4) révèle clairement l'existence d'une antisymétrie par rapport au centre du panneau. Cette transformation s'accommode bien d'une hypothèse de chargement combiné avec cisaillement le long des bords du panneau.

La conjonction de ces observations bien reproduites sur les deux spécimens prospectés et du caractère imprédictible quant à l'apparition des déformations, semble constituer un argument pour avancer un mode 


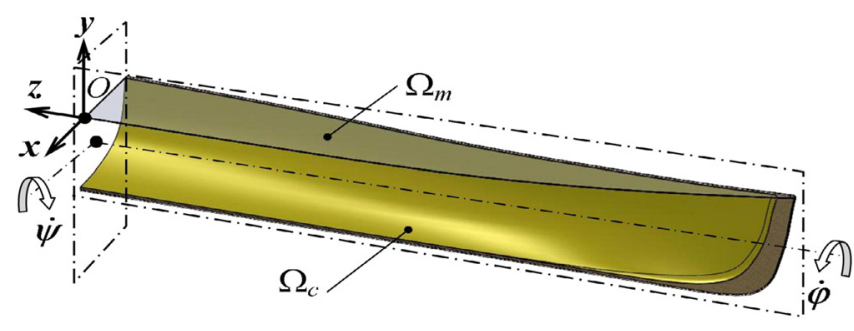

Fig. 5. Restriction du domaine et paramétrage.

de défaillance par voilement. Cette instabilité est vraisemblablement consécutive au refroidissement hétérogène lié à la morphologie du modèle, aux conditions d'échange thermique et aux actions de liaison exercées par le moule sur la coque au niveau de l'interface de contact.

Ces éléments ont donc orienté l'analyse vers une prospection numérique des instabilités lors de la séquence de refroidissement qui n'aurait pas encore suscité des études expérimentales ou numériques.

\section{Formulation du problème d'évolution non-linéaire découplé}

Tenant compte des symétries de la coque par rapport aux plans passant respectivement par la quille et par le maître couple, le domaine $\Omega$ est restreint au quart représenté sur la figure 5 où $\Omega_{\mathrm{m}}$ désigne le moule et $\Omega_{\mathrm{c}} \equiv\left\{\Omega_{i}\right\}$ la coque, l'indice $i=1 ; 3$ référant dans l'ordre aux couches PEHD, mousse PE et PEBD. Les sections d'intersection du domaine avec les plans de symétrie sont désignées par $\partial \Omega_{x}$ et $\partial \Omega_{z}$. Pendant toute la séquence de refroidissement, le système tourne à une vitesse constante $\omega=\dot{\psi} \boldsymbol{x}+\dot{\phi} \boldsymbol{z}$ où les composantes désignent respectivement les vitesses de précession et de rotation propre du système.

Nous référant au processus réel quant au moment d'apparition de l'anomalie, l'étude est limitée à une partie de la phase de refroidissement en prenant pour origine des temps l'instant $t_{0}$ où les fractions cristallisées de tous les polyéthylènes (i.e. PEHD, mousse PE et PEBD) ont atteint leur niveau de saturation. Le système est alors à une température uniforme $T_{0}=100{ }^{\circ} \mathrm{C}$. Le refroidissement est assuré par soufflage de l'interface $\partial \Omega_{0}$ entre le moule et l'ambiance avec un flux d'air à la température $T_{\mathrm{a}}=20^{\circ} \mathrm{C}$. Dans la cavité intérieure mise à la pression atmosphérique par les évents de respiration, la frontière $\partial \Omega_{4}$ du PEBD est soumise à l'action des gaz chauds portés à la température $T_{\mathrm{c}} \approx T_{0}$.

Le principe de mise en œuvre du rotomoulage et la compatibilité physicochimique des phases en présence limitent les risques de délaminage entre les couches. Ceci justifie d'imposer à tout instant la continuité des champs $T$ et $\boldsymbol{u}$ sur l'interface $\partial \Omega_{1-2}$ entre PEHD et mousse et sur l'interface $\partial \Omega_{2-3}$ entre mousse et PEBD. Par contre, la surface de contact unilatéral au niveau de la frontière $\partial \Omega_{01}$ entre le moule et la couche PEHD ne peut s'accommoder de cette continuité notamment en raison de la dilatation différentielle entre la coque et le moule. Ainsi, nous

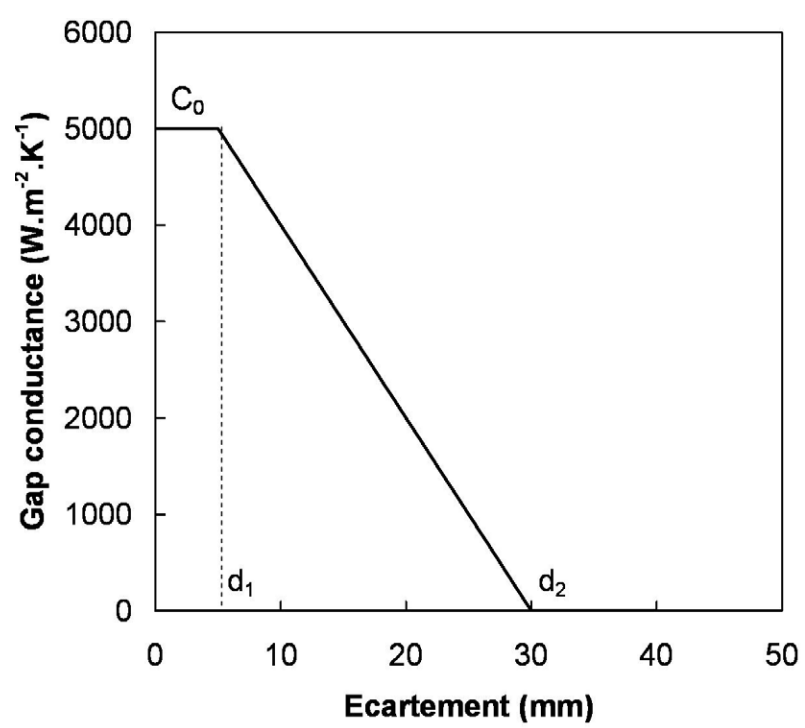

Fig. 6. Modèle gap conductance de l'interface entre moule et peau PEHD.

exprimons la non-linéarité géométrique $\partial \Omega_{0-1} \equiv \partial \Omega_{0-1}$ $(\boldsymbol{u})$ pour rendre compte de l'incidence du décollement sur le transfert de chaleur, sur la rigidité de la structure et sur la distribution du chargement de frottement.

$\mathrm{Au}$ début de la séquence de refroidissement, le contact collant autorise la restriction à un transfert par conduction avec une résistance de contact qui est déterminée plus par la couche en PEHD $\left(k \sim 0,5 \mathrm{~W} \cdot \mathrm{m}^{-1} \cdot \mathrm{K}^{-1}\right)$ que par le moule en aluminium $\left(k \sim 200 \mathrm{~W} \cdot \mathrm{m}^{-1} \cdot \mathrm{K}^{-1}\right)$. À un stade plus avancé, la contraction de la coque s'accompagne d'un mouvement relatif par rapport au moule et de décollements avec infiltration de gaz ou formation d'une poche de vide dans des conditions aléatoires. Ainsi, pour décrire l'évolution des conditions de transfert sur l'interface, nous adoptons dans l'approche proposée le modèle de gap conductance implémenté dans le code Abaqus 6.8 [10]. Les arguments de ce modèle sont la conductance $c_{0}$ à écartement nul, la distance $d_{1}$ de conductance invariable et l'écartement $\left(d_{1}+d_{2}\right)$ au-delà duquel la paroi PEHD est considérée adiabatique. Pour une fonction de raccordement affine par morceaux (Fig. 6), $c_{0}$ est estimée pour une rugosité totale $R_{\mathrm{t}}=100 \mu \mathrm{m} \sim d_{1}$, et la distance $d_{2}$ est déterminée par recalage au regard des réponses $T(t)$ et $\boldsymbol{u}(t)$. Sur cette même interface, les actions de frottement solide sont décrites par le modèle de Coulomb et un coefficient de frottement de glissement $f=0,27$ [11].

Le jeu de données thermophysiques a été constitué par compilation des références [11-15] et à ce propos, il est important de souligner la grande dispersion des valeurs dans la littérature. Les figures $7 \mathrm{a}$ et $\mathrm{b}$ donnent, pour les trois phases, les évolutions de la masse volumique et de la chaleur spécifique à pression constante. On peut remarquer sur la figure $7 \mathrm{~b}$ que dans le sens du chauffage, la transition de phase de second ordre se produit vers $T_{0}$. Le refroidissement relativement lent avec une vitesse moyenne $\dot{T} \approx-0,04 \mathrm{~K} . \mathrm{s}^{-1}$, autorise de négliger l'hystérésis et de considérer la cristallisation achevée. 


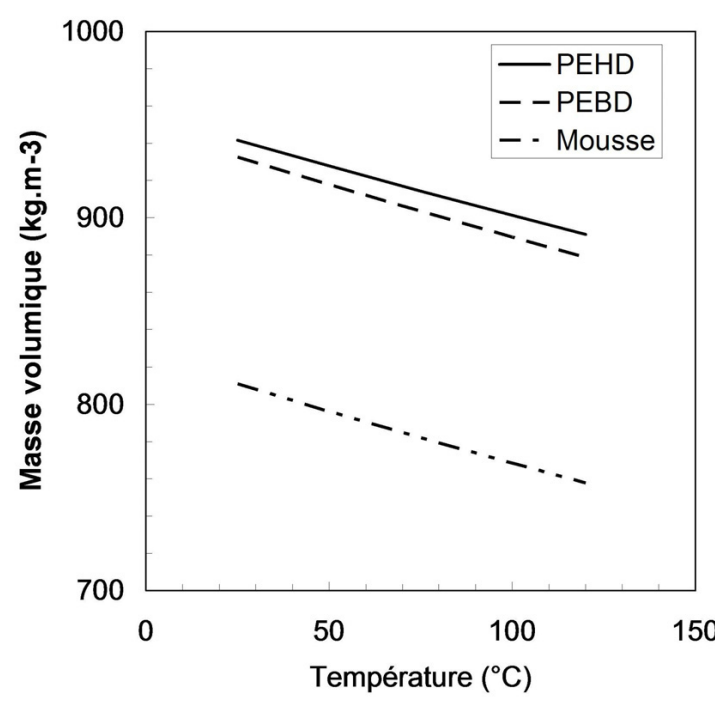

(a)

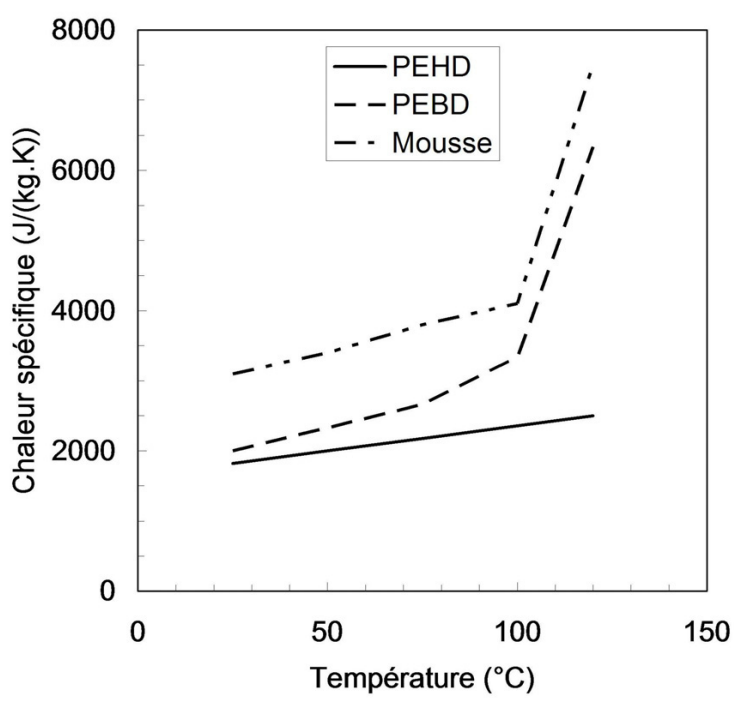

(b)

Fig. 7. Masse volumique et chaleur spécifique des polyéthylènes.

Tableau 1. Propriétés des phases PEHD, PEBD et mousse PET [12-16].

\begin{tabular}{cccc}
\hline Propriété & PEHD & Phase \\
& 0,49 & 0,33 & 0,24 \\
$k\left(\mathrm{~W} \cdot \mathrm{m}^{-1} \cdot \mathrm{K}^{-1}\right)$ & $2,0 \times 10^{-4}$ & $2,5 \times 10^{-4}$ & $2,5 \times 10^{-4}$ \\
$\alpha\left(\mathrm{K}^{-1}\right)$ & 1200 & 300 & 120 \\
$E(\mathrm{MPa})$ & 0,41 & 0,45 & 0,1 \\
$\nu$ & & & \\
\hline
\end{tabular}

Pour la conductivité thermique et le coefficient de dilatation isotrope sur la plage $0{ }^{\circ} \mathrm{C}<T<120{ }^{\circ} \mathrm{C}$ la faible dépendance de la température [14] est un argument pour les admettre invariables. En ce qui concerne les propriétés élastiques $E$ et $\nu$ notamment pour la mousse, on retient des quantités constantes faute de données expérimentales. Ces quantités sont définies par sous-domaine avec, dans le cas de la mousse, des quantités apparentes. Le tableau 1 résume les valeurs adoptées pour ces propriétés.

Dans les conditions de refroidissement appliquées, nous retenons que seul l'échange par convection est significatif. Le coefficient d'échange côté ambiance $h_{\mathrm{a}}$ est évalué pour une convection forcée avec un nombre de Nusselt estimé avec la corrélation empirique de Hilpert [16] $N u=c \operatorname{Re}^{m} \operatorname{Pr}^{1 / 3}$ relative à un cylindre et une vitesse moyenne de l'air de ventilation de $6 \mathrm{~m} . \mathrm{s}^{-1}$. Comme la surface exposée au flux d'air évolue périodiquement et que la géométrie n'est pas cylindrique, la longueur caractéristique dans le nombre de Reynolds est définie par tranche de couples de la coque par sa moyenne sur une période de révolution. On obtient pour $c=0,228$ et $m=0,731$ un coefficient de convection $70<h_{\mathrm{a}}<$ $80 \mathrm{~W} \cdot \mathrm{m}^{-2} \cdot \mathrm{K}^{-1}$ la valeur la plus forte étant rencontrée du côté de l'étrave. Dans la cavité, on considère que le transfert est assuré par convection naturelle avec un coefficient heuristique constant $h_{\mathrm{c}}=5 \mathrm{~W} \cdot \mathrm{m}^{-2} \cdot \mathrm{K}^{-1}$.
On admet qu'à $t_{0}$ le niveau de contrainte hérité suite au changement de phase et au gradient de température est négligeable soit $\sigma_{0} \equiv \sigma\left(\boldsymbol{x}, t_{0}\right)=0$. Par ailleurs, la petitesse de la dissipation intrinsèque et de l'apport de chaleur de changement de phase pour le taux de saturation atteint, permet de découpler les équations de conservation, par l'omission de la source volumique dans l'équation de l'énergie. De même l'apport de chaleur consécutif au frottement solide de la peau PEHD sur le moule est omis. Les vitesses angulaires $\dot{\psi}$ et $\dot{\varphi}$ étant faibles $\left(8\right.$ et $2 \mathrm{~min}^{-1}$ ) on néglige les forces de volume relatives aux actions d'inertie $[17]$.

Pour l'objectif d'analyse en instabilités élastiques, on se place dans le cadre de petites perturbations sauf pour $T$, et on admet pour chaque sous domaine indice $i$ un comportement élastique homogène et isotrope décrit par une loi de Hooke-Duhamel spécifique

$\sigma=\lambda_{i}(\nabla \cdot \boldsymbol{u}) \boldsymbol{I}+\mu_{i}\left(\nabla \boldsymbol{u}+{ }^{t} \nabla \boldsymbol{u}\right)-\alpha_{i} \theta \mathbf{I} \quad$ dans $\Omega_{i}, i=0 ; 3$

Le problème transitoire non-linéaire s'énonce :

Trouver les champs spatio-temporels

$$
\begin{aligned}
& T:\left\{\begin{array}{l}
\left.\left.\Omega \subseteq \varepsilon^{3} \times\right] 0, t_{\mathrm{r}}\right] \rightarrow \mathbb{R} \\
(\boldsymbol{x}, t) \mapsto T(\boldsymbol{x}, t) ; T(\boldsymbol{x}, 0)=T_{0}
\end{array} \quad \text { dans } \Omega\right. \\
& \boldsymbol{u}:\left\{\begin{array}{l}
\left.\left.\Omega \subseteq \varepsilon^{3} \times\right] 0, t_{\mathrm{r}}\right] \rightarrow \mathbb{R}^{3} \\
(\boldsymbol{x}, t) \mapsto \boldsymbol{x}(\boldsymbol{x}, t) ; \boldsymbol{u}(\boldsymbol{x}, 0)=0
\end{array} \text { dans } \Omega\right.
\end{aligned}
$$

vérifiant les équations locales

$$
\nabla \cdot(k \nabla T)=\rho C_{\mathrm{p}} \frac{\partial T}{\partial t} \quad \text { dans } \quad \Omega
$$

$(1-2 \nu) \nabla^{2} \boldsymbol{u}+\nabla(\nabla \cdot \boldsymbol{u})-2 \alpha(1+\nu) \nabla \theta=0 \quad$ dans $\quad \Omega$ 
avec les conditions de frontière

$$
\begin{gathered}
\left\{\begin{array}{l}
-k_{0}(T) \frac{\partial T}{\partial x_{3}}=h_{\mathrm{a}}(\boldsymbol{x})\left(T-T_{\mathrm{a}}\right) \quad \text { sur } \partial \Omega_{0} \\
-k_{3}(T) \frac{\partial T}{\partial x_{3}}=h_{\mathrm{c}}\left(T-T_{\mathrm{c}}\right) \quad \text { sur } \partial \Omega_{4}
\end{array}\right. \\
\begin{cases}u_{x}=0 & \text { sur } \partial \Omega_{x} \\
u_{z}=0 & \text { sur } \partial \Omega_{z} \\
u_{y}=0 & \text { pour } \boldsymbol{x}=0\end{cases} \\
\left\{\begin{array}{l}
\sigma_{31}=-f \mathrm{H}\left(-\sigma_{33}\right)\left|\sigma_{33}\right| \frac{\dot{\boldsymbol{u}}_{1 / 0}(\boldsymbol{x}) \cdot \boldsymbol{x}_{1}}{\left\|\dot{\boldsymbol{u}}_{1 / 0}(\boldsymbol{x}) \cdot \boldsymbol{x}_{1}\right\|} \\
\sigma_{32}=-f \mathrm{H}\left(-\sigma_{33}\right)\left|\sigma_{33}\right| \frac{\dot{\boldsymbol{u}}_{1 / 0}(\boldsymbol{x}) \cdot \boldsymbol{x}_{2}}{\left\|\dot{\boldsymbol{u}}_{1 / 0}(\boldsymbol{x}) \cdot \boldsymbol{x}_{2}\right\|}
\end{array}\right.
\end{gathered}
$$

et respectant la continuité des déformations sur les interfaces $\partial \Omega_{k-(k+1)}$

$$
\left\{\begin{array}{l}
\boldsymbol{x}_{1} \cdot\left(\varepsilon_{k} \cdot \boldsymbol{x}_{1}\right)=\boldsymbol{x}_{1} \cdot\left(\varepsilon_{k+1} \cdot \boldsymbol{x}_{1}\right) \\
\boldsymbol{x}_{2} \cdot\left(\varepsilon_{k} \cdot \boldsymbol{x}_{2}\right)=\boldsymbol{x}_{2} \cdot\left(\varepsilon_{k+1} \cdot \boldsymbol{x}_{2}\right) \\
\boldsymbol{x}_{1} \cdot\left(\varepsilon_{k} \cdot \boldsymbol{x}_{2}\right)=\boldsymbol{x}_{1} \cdot\left(\varepsilon_{k+1} \cdot \boldsymbol{x}_{2}\right)
\end{array}\right.
$$

où $\dot{\boldsymbol{u}}_{1 / 0}$ désigne la vitesse relative du point courant de la coque par rapport au moule et $\varepsilon_{k}, \varepsilon_{k+1}$ les tenseurs de déformation aux points matériels conjugués de l'interface $\partial \Omega_{k-(k+1)}$.

L'analyse du comportement en instabilités élastiques tout au long du processus de refroidissement, est conduite par la méthode de perturbation linéaire qui se ramène à la résolution de l'équation aux valeurs propres

$$
\left(\left[\mathrm{K}_{0}\right]_{t}+\lambda\left[\mathrm{K}_{\Delta}\right]_{t}\right) \cdot \boldsymbol{u}^{*}=0, \forall \boldsymbol{u}^{*} C S A
$$

où $\left[\mathrm{K}_{0}\right]_{t}$ est la matrice de rigidité géométrique de la configuration actuelle du domaine à l'instant courant $t,\left[\mathrm{~K}_{\Delta}\right]_{t}$ la matrice de rigidité différentielle associée à la perturbation et $\boldsymbol{u}^{*}$ est un champ de vitesses virtuelles défini sur $\Omega$. À chaque instant de la séquence de refroidissement, les valeurs propres et les vecteurs propres associés, représentent respectivement les multiplicateurs des charges critiques et les modes de voilement.

\section{Modèle numérique}

Pour résoudre les équations non-linéaires (2), (3) et (8) nous avons utilisé le code de calcul par éléments-finis Abaqus/Explicit. Le recours à un schéma explicite tient plus particulièrement à la gestion de la non-linéarité géométrique de contact liée au décollement et aux actions de frottement.

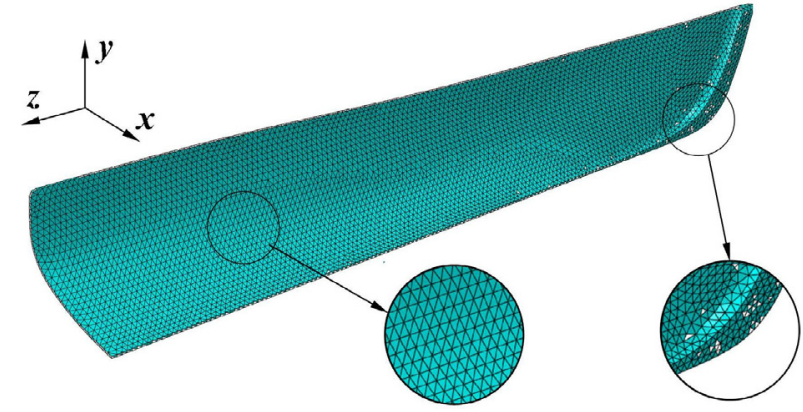

Fig. 8. Maillage de l'âme en mousse de polyéthylène.

Une formulation $3 \mathrm{D}-2 \mathrm{D}$ est retenue pour répondre aux contraintes contradictoires classiques qui prennent une importance particulière dans cette analyse de la stabilité de la structure. Ces contraintes ont trait à la fidélité du modèle géométrique, à la qualité du maillage ainsi qu'aux aspects numériques relatifs à l'adéquation entre la stabilité, la convergence et les ressources de calcul disponibles. D'abord, la localisation du défaut observé dans le voisinage de l'étrave où l'âme de la coque présente de fortes variations d'épaisseur et des courbures importantes justifie une description volumique. Réciproquement, les épaisseurs faibles et assez uniformes des couches PEHD et PEBD s'accommodent bien d'une description surfacique qui tout en permettant l'obtention d'une qualité de maillage satisfaisante (i.e. ratio et aspect) ne pénalise pas la taille du maillage. Par ailleurs, l'assurance de la stabilité du schéma explicite retenu requiert le contrôle de l'incrément de temps maximal ce qui implique une pénalisation par rapport aux ressources nécessaires pour la calcul. La concomitance de ces éléments a conduit à retenir des éléments volumiques pour la mousse ainsi que pour la structure métallique du moule et des éléments surfaciques pour les peaux PEHD et PEBD. La figure 8 représente le maillage construit avec 25500 éléments tétraédriques à 4 nouds pour l'âme en mousse et 5600 éléments hexaédriques à 8 nœuds pour la structure du moule.

La résolution du problème d'évolution et l'analyse de stabilité nécessitent comme l'illustre l'organigramme de la figure 9 , l'actualisation à chaque pas de temps des matrices de capacitance, de masse et de rigidité ainsi que des vecteurs de chargements et flux internes en fonction des réponses $T$ et $\boldsymbol{u}$ au pas précédent. Cette actualisation est dictée par l'évolution des conditions d'échange thermique et de frottement sur l'interface $\partial \Omega_{0-1}(\boldsymbol{u})$ et par l'évolution de la rigidité consécutivement au décollement. Pour une durée $t_{\mathrm{r}}=2000 \mathrm{~s}$, le calcul a nécessité 4 Gø de RAM et près de $100 \mathrm{~h}$ de CPU par simulation.

\section{Résultats et discussions}

La robustesse du modèle posé est étroitement liée à l'ensemble des hypothèses considérées et plus particulièrement à la description heuristique retenue pour le transfert thermique au niveau de l'interface de contact. 


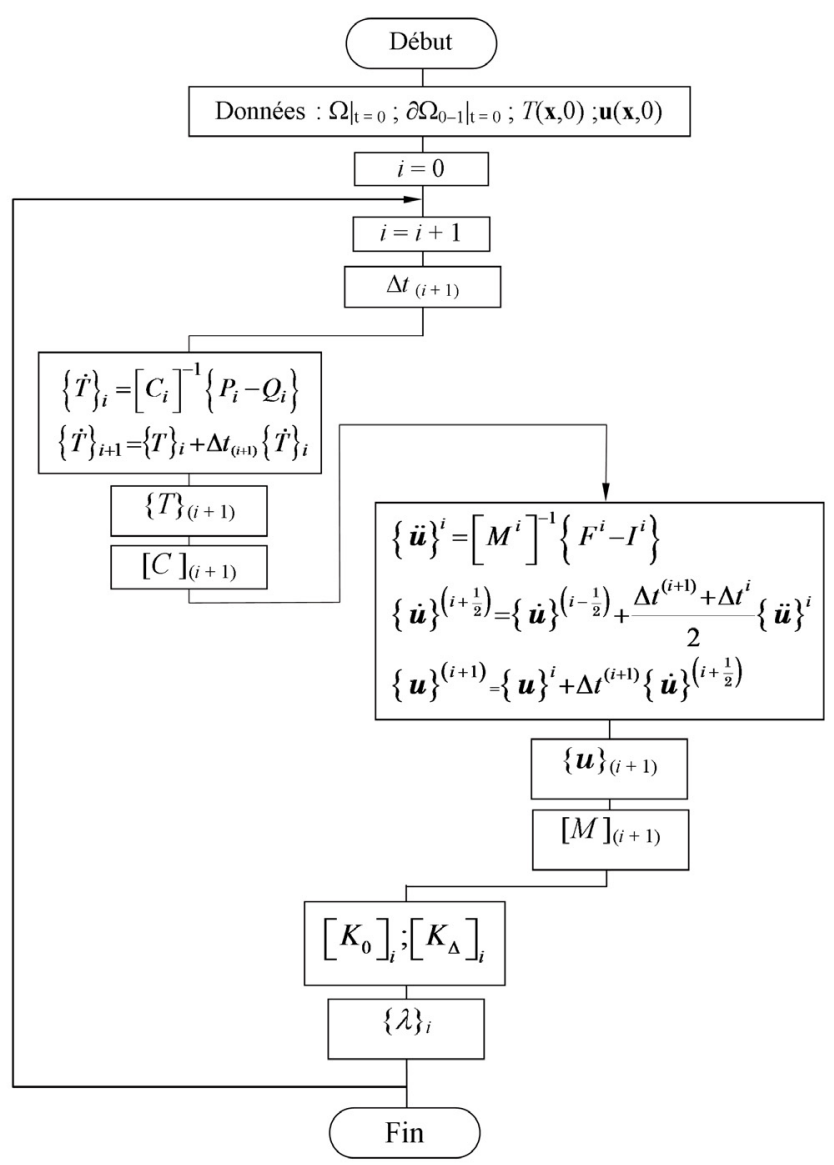

Fig. 9. Organigramme de résolution du problème découplé instationnaire non-linéaire par un schéma explicite.

C'est pourquoi nous avons dans une première étape procédé au recalage du modèle de gap conductance afin d'obtenir une réponse thermique en accord avec une mesure expérimentale in situ. La figure 10 montre, pour différentes valeurs de l'attribut $d_{2}$, les évolutions de la température et du déplacement en un point de référence situé sur l'interface $\partial \Omega_{4}$ dans le voisinage proche du défaut. Pour des valeurs $d_{2}<25 \mathrm{~mm}$, nous avons constaté un réchauffement puis maintien à un palier de température d'autant plus marqué que la valeur est faible alors que pour des valeurs $d_{2} \geq 25 \mathrm{~mm}$ les réponses sont monotones. Pour la valeur $d_{2}=30 \mathrm{~mm}$ retenue dans la suite de l'étude, la réponse en température (Fig. 11) au point de référence (nœud 8205) est en bon accord avec la température mesurée des gaz en contact avec cette paroi.

Les résultats au dernier pas de calcul présentés sur la figure 12 montrent, que malgré les décollements de forte amplitude observés, les surfaces isothermes du moule et de la peau PEHD sont très comparables et présentent toutes deux de faibles gradients. Par contre, la mousse se distingue notamment au niveau de l'étrave, par un niveau de température résiduel proche de $92{ }^{\circ} \mathrm{C}$ et d'un fort gradient selon l'épaisseur. Ces constats semblent appuyer les choix opérés pour la modélisation numérique (i.e. maillage, $T_{0}, t_{\mathrm{r}}, \ldots$ ).

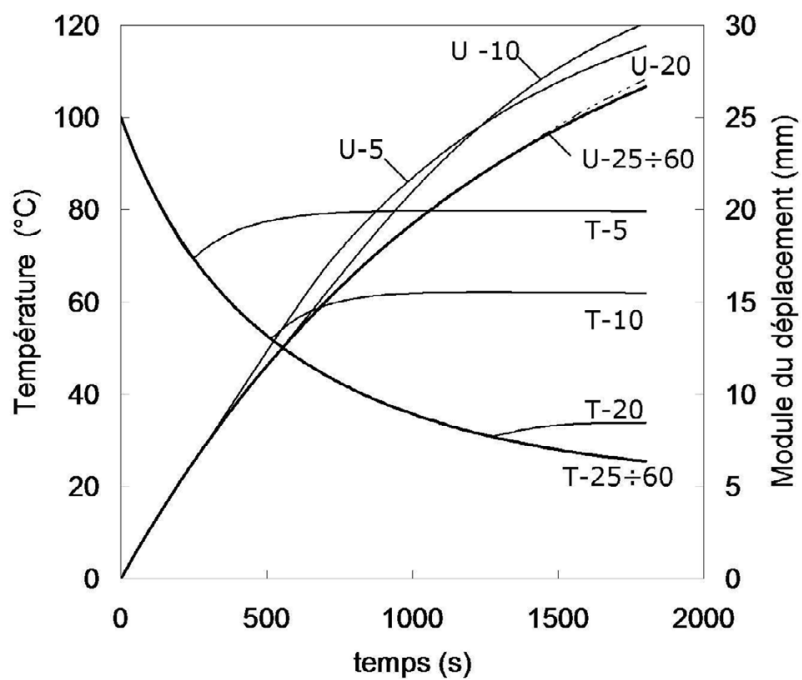

Fig. 10. Recalage de la réponse thermomécanique en fonction de la distance caractéristique $d_{2}$.

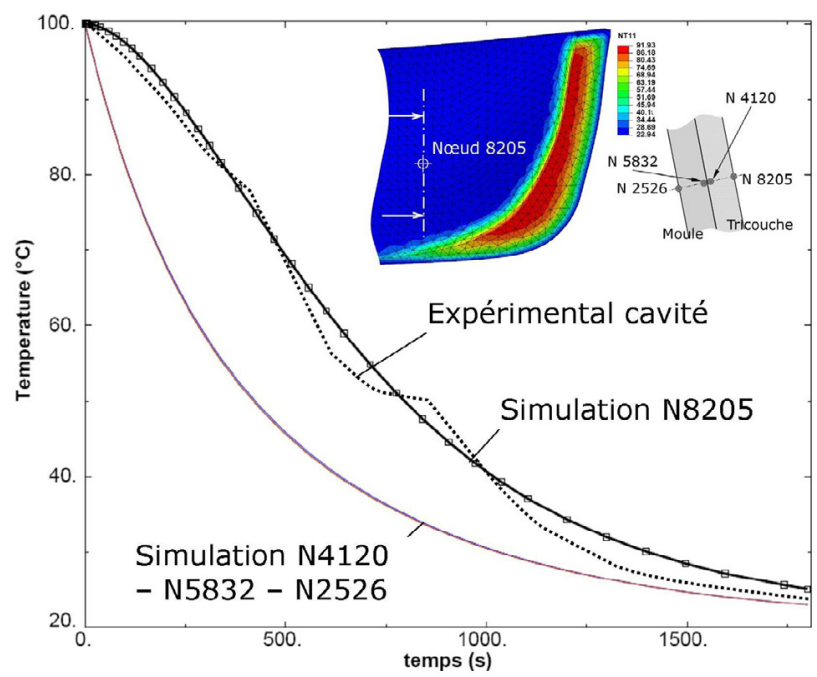

Fig. 11. Réponse en température et comparaison calculs mesure dans la cavité après recalage.

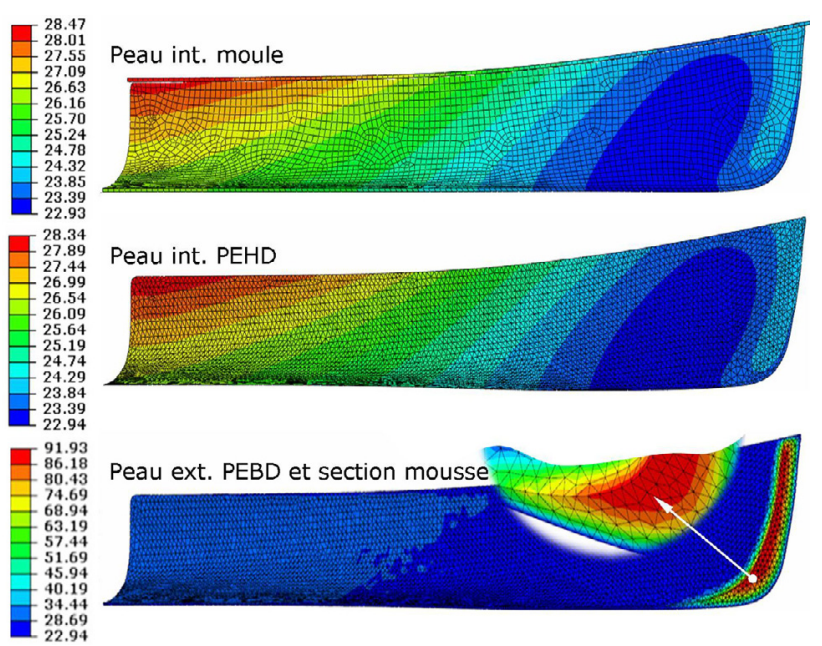

Fig. 12. Champs de température au dernier pas de calcul. 

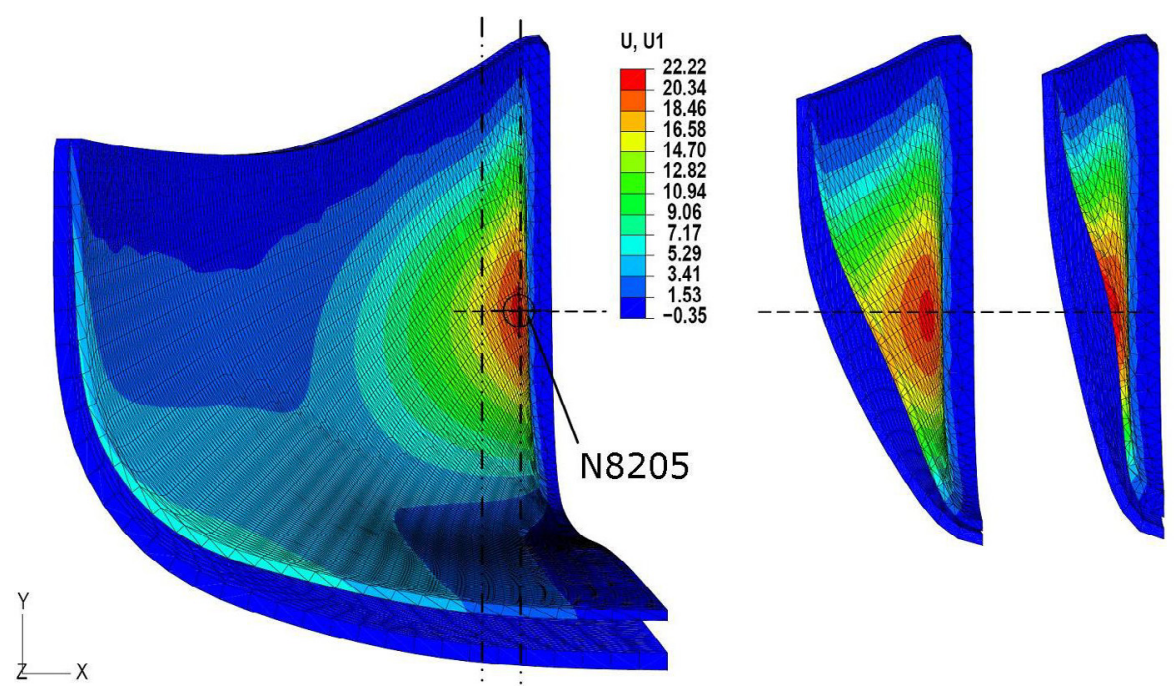

Fig. 13. Déplacement $u_{x}$ et décollement au dernier pas de calcul. Vue selon l'axe de la coque.
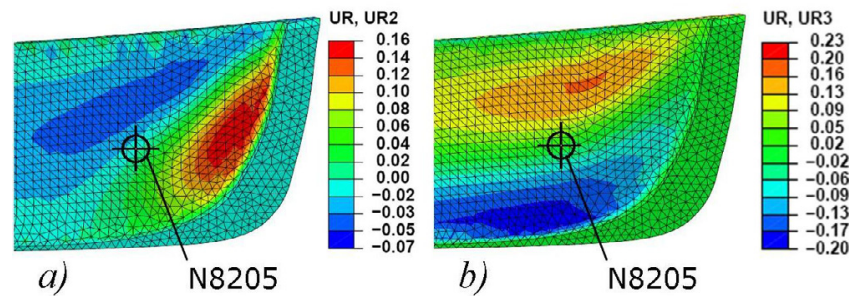

Fig. 14. Antisymétrie des rotations au voisinage de l'étrave au dernier pas de calcul.

Les simulations en thermoélasticité ont permis de localiser les lieux de décollement sur le bordé de coque. Les amplitudes les plus importantes se manifestent d'une part au niveau du fond du bordé de coque où la rigidité en flexion est relativement faible et d'autre part dans la zone d'apparition de l'anomalie où l'on s'attendait à des amplitudes plus réduites vue la rigidité augmentée par la présence de l'étrave. On remarque sur les isovaleurs de la composante de déplacement normal données par la figure 13, que le nœud retenu pour la qualification de la solution thermique est situé dans la zone où l'amplitude du décollement atteint un maximum d'environ $22 \mathrm{~mm}$.

Ce décollement dans la zone de l'anomalie est vraisemblablement provoqué par une flexion bidirectionnelle de la coque sous l'effet du moment d'encastrement avec le couvercle du moule, et par le blocage des rotations au niveau de l'étrave très rigide en flexion. Cette interprétation est cohérente avec l'antisymétrie des composantes de rotation illustrées respectivement par les figures $14 \mathrm{a}$ et b. On peut remarquer aussi que le nœud retenu appartient dans les deux directions aux zones où le changement de signe se manifeste. Ceci conforte l'assertion d'existence d'une symétrie centrale pour la configuration déformée.

L'analyse de la stabilité élastique a été limitée aux 5 premiers modes et a couvert toute la séquence de refroidissement. Pour tous les pas de temps, les 2 premiers modes de voilement sont localisés au voisinage de l'étrave
(Fig. 15) alors que tous les modes d'ordre supérieur apparaissent au milieu de la coque sur le bordé de fond. Le coefficient critique du premier mode pour le dernier pas de refroidissement est $\lambda_{\mathrm{c} 1}=0,33$ et ne change que légèrement d'un pas à un autre. Le deuxième mode s'écarte du premier avec $\lambda_{\mathrm{c} 2}=0,40$ ce qui semble expliquer la prépondérance d'apparition du premier mode observée dans la pratique. Pour le mode 2, l'antisymétrie des rotations et des déplacements est bien confirmée dans les trois directions comme le montrent les figures 16a et b où on représente les isovaleurs de ces champs sur la peau PEHD au dernier pas de calcul. Les 3 modes suivants se manifestent avec des coefficients critiques de 0,43-0,510,53 . L'importance décroissante de ces modes conduit à des amplitudes de déplacement de plus en plus faibles et les défauts qu'ils engendrent peuvent rester dans les limites d'acceptation du produit.

Notons que par rapport à une pratique des rotomouleurs qui consiste à réaliser un polissage de la surface active du moule, nous avons procédé à des simulations avec différentes valeurs de rugosité. Les résultats n'ont pas montré une influence notable de ce facteur sur la réponse en voilement ce qui remet en question cette pratique dès lors qu'on la préconise pour limiter les risques de déformation.

\section{Conclusion}

L'étude de cas présentée a montré qu'un risque réel d'instabilité est encouru pendant la séquence de refroidissement et le modèle proposé semble offrir une robustesse au niveau de :

1. la réponse thermique qui rejoint la mesure expérimentale dans la cavité;

2. la prise en compte de la non-linéarité géométrique qui conduit à des décollements compatibles avec l'observation; 

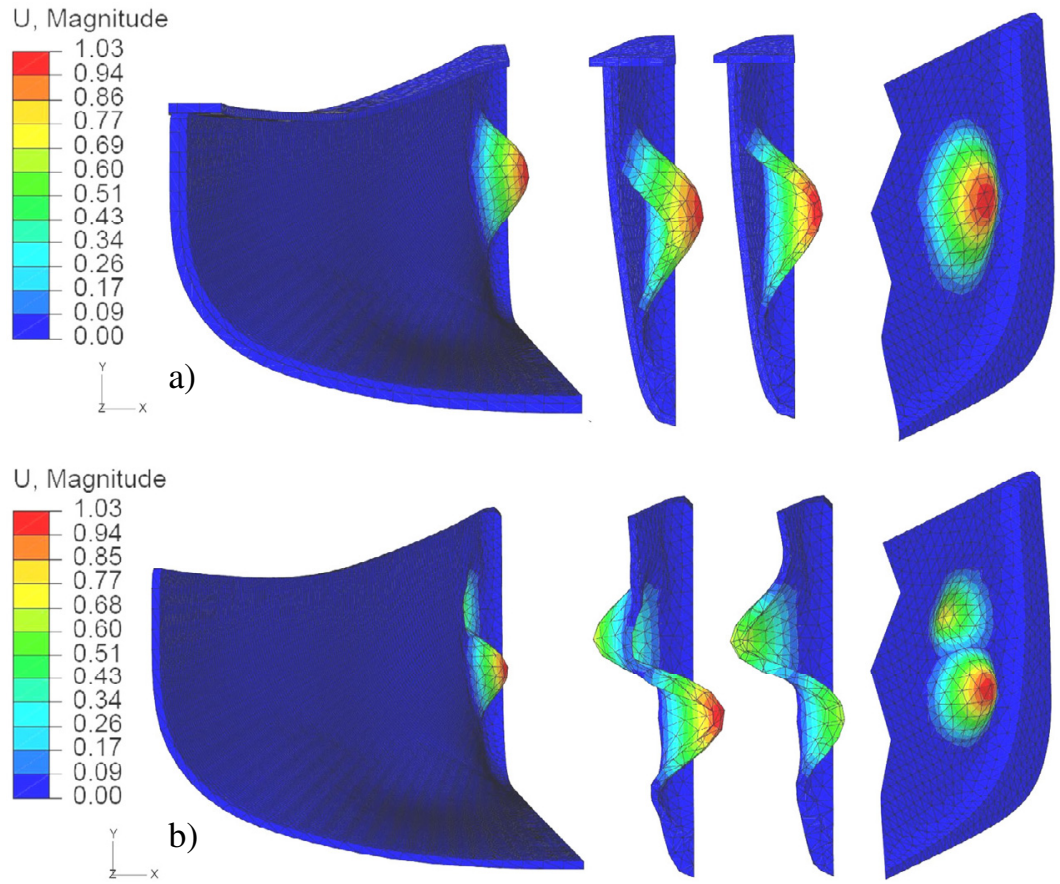

Fig. 15. Modes de voilement. (a) - Mode 1 ; (b) - mode 2.
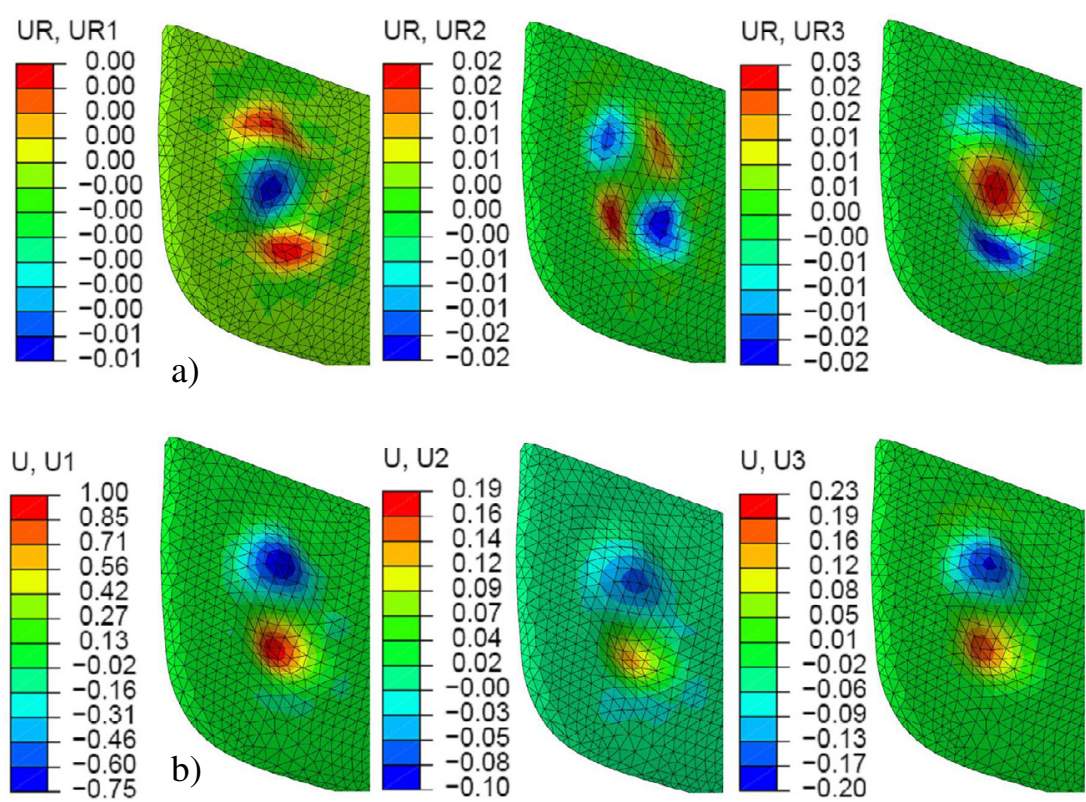

Fig. 16. Champs des composantes de rotation et de déplacement du mode 2 dans les 3 directions. (a) - Rotations; (b) - déplacements.

3. la nature, la localisation et l'ordre d'apparition des modes de voilement qui restituent bien les défauts observés.

Le niveau de concordance entre le premier mode d'instabilité calculé et la géométrie observée traduit l'importance de la séquence de refroidissement pour la prédiction de la géométrie finale de la pièce. En effet, les résultats obtenus semblent confirmer la prédominance des mécanismes qui gouvernent l'évolution lors du refroidissement et par suite la suffisance de la restriction admise à cette seule séquence.

Malgré la limitation à une formulation en élasticité linéaire et bien que l'analyse soit restreinte à l'intervalle de temps où toutes les phases sont totalement cristallisées, le modèle proposé devrait aider à une meilleure compréhension des causes de certaines défaillances qui continuent à pénaliser les processus par rotomoulage. 


\section{Références}

[1] R.J. Crawford, Rotational molding of plastics 2nd edition, Research studies press, England, 1996

[2] S.F. Bush, O.K. Ademosu, Low-density rotomoulded polymer foams, Colloids and Surfaces A: Phys. Eng. Aspects 263 (2005) 370-378

[3] D. Gay, Matériaux composites, Lavoisier, Paris, 2005

[4] A. Tcharkhtchi, Rotomoulage de pièces en matière thermoplastique, Les techniques de l'ingénieur, traité plasturgie AM3706, 2008

[5] G. Kalyanjit, Thermal models and energy saving strategies for rotational molding operations, Thesis, Georgia Institute of Technology, 2004

[6] C.T. Bellehumeur, Polymer sintering and its role in rotational molding, Ph.D., McMaster University, Hamilton, 1997

[7] E. Pérot, Optimisation et modélisation du procédé de rotomoulage, Thèse, École doctorale des matériaux de Lyon, Lyon, 2006

[8] R. Le Goff, G. Poutot, D. Delaunay, R. Fulchiron, E. Kocher, Study and modeling of heat transfer during the solidification of semi-crystalline polymers, Int. J. Heat Mass Trans. 48 (2005) 5417-5430

[9] Commissariat à l'énergie atomique CEA - DEN/DM2S/ SEMT

[10] Abaqus Theory Manual version 6.8, Simulia-Dassault Systèmes, 2008

[11] N. Rao, K. O'Brien, Design Data for Plastics Engineers, Hanser Publishers, Munich, 1998

[12] D.V. Rosato, M.G. Rosato, Plastics design handbook, Kluwer Academic Publishers, Boston/Dordrecht/ London, 2001

[13] J.A. Brydson, Plastics materials, 7th edition, Butterworth-Heinemann, Oxford, 1999

[14] Data base of materials, CosmosWorks, 2008

[15] M.L. Berins, Plastics engineering handbook of the society of the plastics industry, Kluwer Academic Publishers, Boston/Dordrecht/London, 1991

[16] F.P. Incropera, D.P. De Witt, Fundamentals of heat and mass transfer, John Wiley \& Sons, New York, 1996

[17] R.J. Crawford, J.L. Throne, Rotational molding technology, William Andrew Publishing, New York, 2002 